\title{
COVID-19 patient plasma demonstrates resistance to TPA-induced fibrinolysis as measured by thromboelastography
}

\author{
Cheryl L. Maier ${ }^{1} \cdot$ Tania Sarker $^{1}$ (D) $\cdot$ Fania Szlam $^{2} \cdot$ Roman M. Sniecinski $^{3}$ (D)
}

Accepted: 26 March 2021 / Published online: 7 April 2021

(c) The Author(s), under exclusive licence to Springer Science+Business Media, LLC, part of Springer Nature 2021

\begin{abstract}
Patients critically ill with COVID-19 are at risk for thrombotic events despite prophylactic anticoagulation. Impaired fibrinolysis has been proposed as an underlying mechanism. Our objective was to determine if fibrinolysis stimulated by tissue plasminogen activator (tPA) differed between COVID patients and controls. Plasma from 14 COVID patients on prophylactic heparin therapy was obtained and compared with heparinized plasma from 14 different healthy donors to act as controls. Kaolin activated thromboelastography with heparinase was utilized to obtain baseline measurements and then repeated with the addition of $4 \mathrm{nM}$ tPA. Baseline fibrinogen levels were higher in COVID plasma as measured by maximum clot amplitude $(43.6 \pm 6.9 \mathrm{~mm}$ vs. $23.2 \pm 5.5 \mathrm{~mm}, \mathrm{p}<0.0001)$ and Clauss assay $(595 \pm 135 \mathrm{mg} / \mathrm{dL}$ vs. $278 \pm 44 \mathrm{mg} / \mathrm{dL}, \mathrm{p}<0.0001)$. With the addition of tPA, fibrinolysis at $30 \mathrm{~min}$ after MA (LY30\%) was lower $(37.9 \pm 16.5 \%$ vs. $58.9 \pm 18.3 \%, \mathrm{p}=0.0035)$ and time to $50 \%$ lysis was longer $(48.8 \pm 16.3$ vs. $30.5 \pm 15.4 \mathrm{~min}, \mathrm{p}=0.0053)$ in the COVID- 19 samples. Clotting times and rate of fibrin polymerization (' $R$ ' or ' $\alpha$ ' parameters) were largely the same in both groups. Clot from COVID patients contains a higher fibrin content compared to standard controls and shows resistance to fibrinolysis induced by tPA. These findings suggest the clinical efficacy of thrombolytics may be reduced in COVID-19 patients.
\end{abstract}

Keywords Thromboelastography · Fibrinolysis · COVID-19 - Severe acute respiratory syndrome coronavirus 2 . Tissue plasminogen activator

\section{Highlights}

- The fibrinogen content of COVID-19 patients is significantly higher than healthy controls

- The density of fibrin in COVID-19 thrombus is likely higher than in other patients

- COVID-19 thrombus is less susceptible to tPA-induced fibrinolysis

- The appropriate dosing for using fibrinolytic agents in COVID-19 thrombosis needs to be investigated

Roman M. Sniecinski

rsnieci@emory.edu

1 Department of Pathology and Laboratory Medicine, Emory University School of Medicine, Atlanta, GA, USA

2 Department of Anesthesiology, Emory University School of Medicine, Atlanta, GA, USA

3 Department of Anesthesiology, Emory University Hospital, Emory University School of Medicine, 3rd Floor, 1364 Clifton Rd, NE, Atlanta, GA 30322, USA

\section{Introduction}

Patients with COVID-19, the disease caused by the severe acute respiratory syndrome coronavirus 2 (SARS-CoV-2), are at increased risk for venous thrombotic events (VTE) despite prophylactic anticoagulation [1]. The hypercoagulability profile is characterized by very high fibrinogen and d-dimer levels, elevated Factor VIII and von Willebrand factor levels, as well as reduced measurements of natural anticoagulants such as Protein C, Protein S, and antithrombin [2]. Viscoelastic testing (i.e. thromboelastography (TEG) or rotational thromboelastometry) typically demonstrates increased clot strength with a higher fibrin contribution [3-5].

Perhaps somewhat paradoxically in light of their elevated d-dimers, COVID-19 patients are also thought to exhibit reduced fibrinolytic activity. "Fibrinolytic shutdown" as defined by viscoelastic testing has been reported in these patients and is associated with an increased incidence of VTE $[6,7]$. It has been suggested that fibrinolytic agents may be a therapeutic option to treat this impaired fibrinolysis 
[7]. Micro clots in the lungs may also be important in the development of sepsis-related acute respiratory distress syndrome (ARDS). In a phase I clinical trial in trauma patients, Hardaway et al. demonstrated improved $\mathrm{PaO} 2$ with streptokinase administration in patients suffering from ARDS, presumably from dissolution of these micro clots [8]. This reasoning formed the basis for a recent small case series of three intubated COVID-19 patients with ARDS treated with low dose tissue plasminogen activator (tPA) in which improvement of $\mathrm{P} / \mathrm{F}$ ratios were observed [9].

Given the increased clot strength described in COVID19 , it is possible that the effects of fibrinolytic therapy in these patients may be reduced. We aimed to compare the fibrinolytic response of tPA using TEG measurements on patient and control plasma. We hypothesized that COVID-19 patients would show reduced clot lysis percentage compared to controls.

\section{Materials and methods}

\section{Sample collection}

Platelet poor plasma (PPP) was obtained from patients diagnosed with acute COVID-19 and admitted to Emory University Hospital between 6/28/2020 and 7/11/2020. All samples were collected within 8 days of admission, Any associated clinical labs were collected in a deidentified fashion according to a protocol approved by Emory's Institutional Review Board. All patients were on prophylactic anticoagulation with either low molecular weight or unfractionated heparin at the time of collection. Patients on any other anticoagulation medications were excluded. Plasma from 14 different healthy individuals ( 7 male and 7 female) was purchased (George King Bio-Medical Inc., Overland Park, KS) to act as controls. Prior to testing, unfractionated heparin $(1.5$ units $/ \mathrm{mL})$ was added to each control sample to replicate patient anticoagulation. All samples were stored at $-80{ }^{\circ} \mathrm{C}$ until thawed for testing.

\section{Thromboelastography}

All testing was performed on the TEG 5000 (Haemonetics, Boston, MA) using heparinase cups containing 2.0 IU lyophilized Heparinase I, which is capable of neutralizing heparin levels of up to $6.0 \mathrm{u} / \mathrm{mL}$ according to the manufacturer. This has been previously shown to neutralize both unfractionated and low molecular weight heparins (LMWH) for TEG testing [10]. Patient and control plasma were tested both with and without the addition of tPA (final concentration $4 \mathrm{nM}$, Activase alteplase, Genentech, San Francisco, CA) to the cup.
Our primary measurements of interest were those TEG parameters related to fibrinolysis. The Ly30 and Ly60 parameters represent the percentage decrease in amplitude that occurs 30 and 60 min respectively after the maximum amplitude (MA) is reached [11]. The higher the percentage, the greater amount of fibrinolysis has taken place. The MA, measured in millimeters, represents clot strength and is normally a function of platelet count and fibrinogen interactions, with higher levels of each associated with greater clot amplitude. However, when PPP is used, the clot amplitude is dependent only on fibrinogen interactions. The clot lysis time (CLT) is another parameter of fibrinolysis and represents the elapsed time from when MA is reached to $50 \%$ clot lysis. Although not related to fibrinolysis, we collected the $\mathrm{R}$ time (R), which is a clotting time that begins with the addition of calcium and kaolin to the citrated sample and ends with initial fibrin formation. The alpha angle $(\alpha)$, which is a function of fibrin polymerization rate, was also recorded.

\section{Statistical analysis}

A convenience sample of COVID-19 patients was planned. Normality of the data was assessed using the Kolmogorov-Smirinov test and visual inspection of Q-Q plots. Unpaired $t$ tests or Wilcoxon's exact test as appropriate were used to compare continuous variables between groups. Probability values of $\alpha<0.05$ were required to reject the null hypothesis in a two-tailed test. All calculations were made using SAS 9.4 (SAS Institute, Cary, NC) and Prism 9.0 (GraphPad Software, San Diego, CA).

\section{Results}

Samples were collected from 14 different patients, 8 of whom were admitted to the ICU and 6 of whom were on the hospital ward floor. All samples were collected within 8 days of admission (range 1-8). Floor patients were exclusively on LMWH anticoagulation while the majority of ICU patients were on infusions of unfractionated heparin. Available laboratory results for these patients are provided in Table 1 . Fibrinogen by Clauss assay was also measured in the 14 samples from healthy controls. Samples from COVID-19 patients contained approximately twice as much fibrinogen $(594 \pm 135$ vs. $278 \pm 44 \mathrm{mg} / \mathrm{dL}, \mathrm{p}<0.001)$.

The TEG measurements with and without the addition of tPA are presented in Table 2. Like the Clauss fibrinogen level, the MA parameter was also twice as high in COVID19 samples. The $\mathrm{R}$ was also longer in the COVID-19 plasma. Neither COVID-19 nor control plasma showed any fibrinolysis at $30 \mathrm{~min}$ in the standard heparinase cups and thus LY60 and CLT measurements were not obtained. 
Table 1 Anticoagulation and standard laboratory tests of COVID-19 patients with breakdown of those patients in the ICU vs. on the floor

\begin{tabular}{lllll}
\hline & Total cohort & ICU patients & Floor patients & $\mathrm{p}$ \\
\hline $\mathrm{N}$ & 14 & 8 & 6 & - \\
UFH infusion (vs. LMWH) & $5(36 \%)$ & $5(63 \%)$ & $0(0 \%)$ & 0.0310 \\
d-dimer level (ng/mL) & $1152(710,2002)$ & $1154(804,1963)$ & $1045(681,4567)$ & 0.5251 \\
C-reactive protein (mg/L) & $105.0 \pm 85.6$ & $143.7 \pm 93.9$ & $53.5 \pm 33.0$ & 0.0324 \\
Fibrinogen (mg/dL) & $594 \pm 135$ & $646 \pm 104$ & $527 \pm 150$ & 0.1045 \\
\hline
\end{tabular}

Continuous variables are presented as mean \pm standard deviation or median (1st quartile, 3rd quartile) based upon normality of the data. Categorical variables are presented as $\mathrm{n}(\%)$. Unpaired $t$ tests were utilized in the comparison between ICU and floor patients for C-reactive protein and fibrinogen levels. Wilcoxon's exact test was used to evaluate the hypothesis that d-dimer levels were higher in ICU than floor patients. Fisher's exact test was utilized to compare the proportion of patients on heparin infusions instead of LMWH between ICU and floor patients

$U F H$ unfractionated heparin, $L M W H$ low molecular weight heparin
Table 2 TEG measurements of control and COVID-19 plasma at baseline and with the addition of $4 \mathrm{nM}$ tPA

\begin{tabular}{|c|c|c|c|}
\hline & Control $(n=14)$ & COVID-19 $(n=14)$ & $\mathrm{p}$ \\
\hline \multicolumn{4}{|l|}{ Heparinase TEG } \\
\hline $\mathrm{R}(\min )$ & $5.5 \pm 1.3$ & $6.8 \pm 1.9$ & 0.0429 \\
\hline$\alpha$ angle $\left({ }^{\circ}\right)$ & $67.8 \pm 8.7$ & $71.4 \pm 10.5$ & 0.3325 \\
\hline MA (mm) & $24.3 \pm 5.4$ & $51.1 \pm 11.0$ & $<0.001$ \\
\hline $\begin{array}{l}\text { Lysis at } 30 \mathrm{~min} \\
(\%)\end{array}$ & $0 \pm 0$ & $0 \pm 0$ & - \\
\hline \multicolumn{4}{|c|}{ Heparinase TEG with 4 nM tPA } \\
\hline $\mathrm{R}(\min )$ & $6.1 \pm 1.6$ & $6.9 \pm 2.0$ & 0.2230 \\
\hline$\alpha$ angle $\left({ }^{\circ}\right)$ & $68.5 \pm 7.9$ & $73.5 \pm 5.3$ & 0.0599 \\
\hline MA (mm) & $23.2 \pm 5.5$ & $43.6 \pm 6.9$ & $<0.0001$ \\
\hline $\begin{array}{l}\text { Lysis at } 30 \mathrm{~min} \\
(\%)\end{array}$ & $58.9 \pm 18.3$ & $37.9 \pm 16.5$ & 0.0035 \\
\hline $\begin{array}{l}\text { Lysis at } 60 \mathrm{~min} \\
(\%)\end{array}$ & $77.5 \pm 11.4$ & $62.4 \pm 15.4$ & 0.0067 \\
\hline CLT (min) & $30.5 \pm 15.4$ & $48.8 \pm 16.3$ & 0.0053 \\
\hline
\end{tabular}

TEG was performed on thawed plasma using kaolin as an activator along with heparinase both with and without the addition of $4 \mathrm{nM}$ human tPA (Activase ${ }^{\circledR}$, Genentech, San Francisco, CA). Results presented as mean \pm standard deviation. No lysis was evident at $30 \mathrm{~min}$ in samples without tPA, so no further measurements (LY60, CLT) were taken. Statistical comparisons were made using unpaired $t$ tests

Both groups showed a marked increase in LY30 and Ly60 with the addition of tPA. The mean amount of clot lysis in COVID-19 patients was $21 \%$ less than controls at $30 \mathrm{~min}$ and $15 \%$ less at $60 \mathrm{~min}$. The mean CLT was more than $50 \%$ longer in COVID-19 samples compared to controls All fibrinolysis measurements are presented graphically in Fig. 1. For other TEG parameters, only the MA was significantly higher in the COVID-19 samples.

\section{Discussion}

COVID-19 patients are known to display a hypercoagulable profile on whole blood TEG consistent with increased clot strength and high fibrinogen levels [12]. Our sample of patients included ICU and hospital ward floor who, by C-reactive protein measurements, would be classified as having at least moderate or severe disease [13]. Since our plasma samples contained no platelets, the measured clot strength in this study was entirely dependent on fibrin interactions. The higher MA measured in COVID-19 samples both with and without tPA addition was expected. This was also confirmed with the baseline differences in fibrinogen measurements by Clauss assay, which is consistent with previously reported hematological findings of this disease.

Our primary aim was not to demonstrate increased fibrinogen levels, but to investigate the effects of tPA on fibrinolysis in COVID-19 patients. However, the optimal dose to use for this purpose in plasma-based TEG is unclear. In the trauma literature, Moore et al. have utilized doses of $75 \mathrm{ng} /$ $\mathrm{mL}(\sim 1.1 \mathrm{nM})$ for "tPA challenge TEG" in whole blood, which would be equivalent to approximately $2 \mathrm{nM}$ in plasma [14]. Ostrowski et al. utilized $1.8 \mathrm{nM}$ tPA when comparing plasma from patients with the human immune-deficiency virus (HIV) to controls [15]. They obtained Ly30 values of $24.2 \%$ in healthy controls (vs. $53.9 \%$ in hypocoagulable HIV samples) [15]. Given the hypercoagulable profile of COVID-19, we were not sure if these previously reported doses would show any effect, so we decided to use $4 \mathrm{nM}$ tPA, which was about twice the concentration of these prior studies. Like Ostrowski et al., we saw no fibrinolysis on the standard TEG in either group. We did obtain about twice their reported Ly30 values in controls with the addition of the $4 \mathrm{nM}$ tPA, which would seem to be reasonable given the dose was twice as high. We are thus confident that our controls and tPA dosing were appropriate for this study.

Decreased fibrinolysis in COVID-19 patients was also recently reported by Bachler et al. using whole blood on 
Fig. 1 Graphs demonstrate the fibrinolysis parameters of Ly30 and Ly60 on top and CLT on the bottom comparing control (filled circles) and COVID-19 (filled squares) measured on heparinase TEG with the addition of $4 \mathrm{nM}$ tPA. Bar graphs depict the mean of each measurement (light grey is control, with dark grey COVID-19) and $95 \%$ confidence intervals. Unpaired $t$ tests were used to compare each parameter, with $\mathrm{p}$ values noted

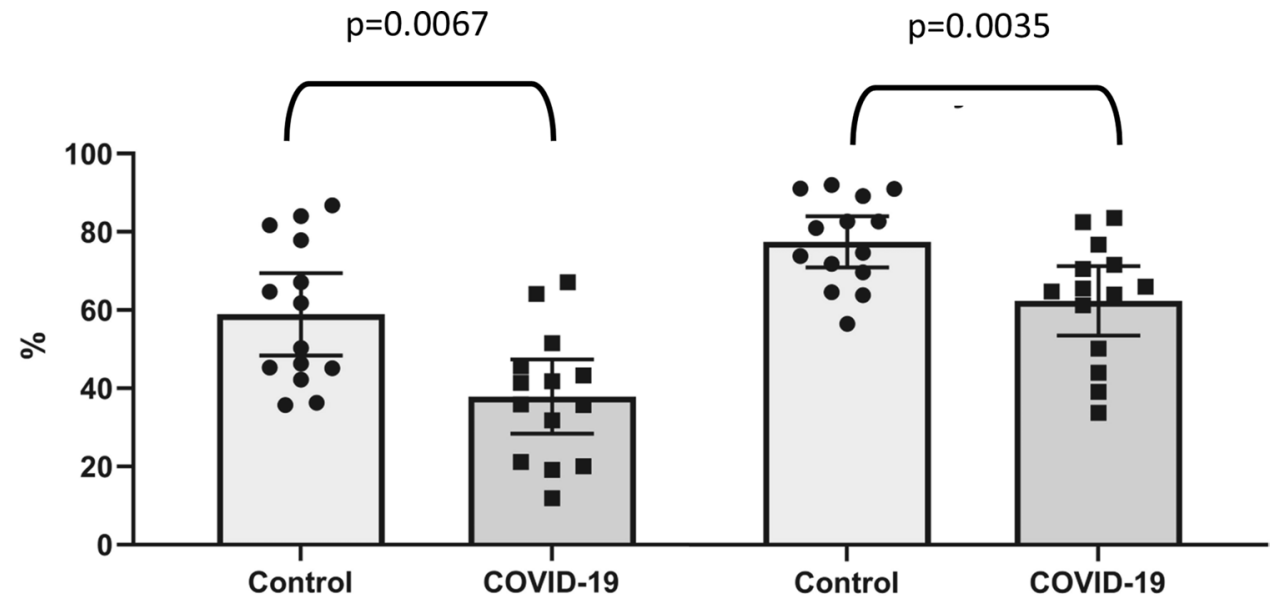

LY 30

LY 60

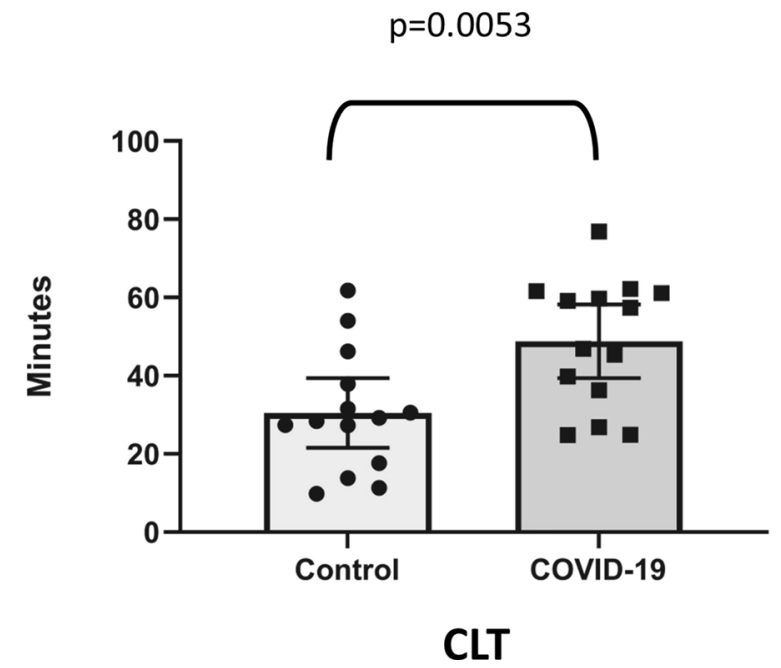

the Clot Pro (Enicor GmbH, Munich, Germany) viscoelastic platform [16]. They reported a more than doubled lysis time (LT) in COVID-19 patients compared to healthy controls. The LT parameter is very similar to the TEG CLT parameter that we measured, although the dose of tPA in the Clot Pro is approximately 2.5 times higher than what was used in our study. While it is difficult to translate values across different viscoelastic testing platforms, it is notable that our study in plasma demonstrated about the same magnitude of resistance to fibrinolysis as Bachler et al.

There are several possibilities for the reduced fibrinolysis demonstrated in the COVID-19 samples. One plausible explanation is that there are increased levels of fibrinolytic inhibitors present. Plasminogen activator inhibitor-1 (PAI1), for example, has been shown to be elevated in these patients [17]. The mechanism is thought to be due to the downregulation of angiotensin converting enzyme by the coronavirus (SARS-CoV-2), leading to increased levels of angiotensin II, an inducer of PAI-1 in endothelial cells [18].
Another possibility may simply be the high fibrinogen levels lead to clot formation with a dense fibrin structure, making it less susceptible to tPA-induced fibrinolysis [19]. Increased fibrinogen in COVID-19 patients has been shown to correlate with levels of the inflammatory cytokine IL-6, which is also associated with more severe disease [20,21]. Recently, our group reported on therapeutic plasma exchange in six critically ill COVID-19 patients which reduced their fibrinogen levels by about of $50 \%$ [22]. Notably, the fibrinogen levels remained low and four of the six patients had clinical improvement. It is possible that simply reducing fibrinogen levels may decrease the sequalae of COVID-19 hypercoagulability. By decreasing clot strength within the microvasculature, the natural actions of the fibrinolytic system may be more effective.

Despite the high fibrinogen levels, our study did not demonstrate significantly different values for the $\alpha$ angle between COVID-19 and control plasma. This perhaps suggests that the inhibition of fibrinolysis may be more important than 
an increased rate of fibrin formation in the development of COVID-19 hypercoagulability. Although there was a difference in R between COVID-19 and control plasma at baseline, this was not reproduced in the TEGs with TPA addition. In general, COVID-19 patients are not thought to have exceptionally prolonged clotting times [5], and it is possible the prolonged $\mathrm{R}$ observed on baseline measurements was simply due to chance or perhaps less effective heparin neutralization in that particular batch of TEG cups.

This was a small ex-vivo study with some obvious limitations. Other than being in the ICU or on the wards, we were not able to collect much demographic information given that the samples were deidentified. It is possible that other comorbidities in addition to COVID-19 existed in these patients that could have increased their thrombogenicity and resistance to clot lysis. Additionally, since it was performed in platelet-poor plasma, the TEG measurements only reflect fibrinogen contributions to clot strength, ignoring the role of platelets. Some studies have suggested that platelets can enhance clot resistance to fibrinolysis [23]. Prior whole blood viscoelastic studies in COVID-19 patients have shown an increased platelet contribution to clot strength, but this still seems to be less prominent than the fibrinogen contribution [20]. Some authors have reported increased aggregation in COVID-19 platelets possibly due to increased thromboxane generation [24]. However, aspirin does not appear to prevent COVID-19 thrombosis [25]. Further studies are warranted to investigate the role of platelets in COVID-19 hypercoagulability.

In conclusion, we have demonstrated an increased resistance to tPA-induced fibrinolysis in COVID-19 plasma. This may be due, at least in part, to simply having a higher fibrinogen content, although other mechanisms are possible. The reduced efficacy of tPA may have clinical implications. As described in a recent case report, COVID-19 clot dissolution can require more than $100 \mathrm{mg}$ of tPA and catheterdirected treatment as well [26]. Typical dosing schemes of fibrinolytics may need to be increased, but further research is required.

Author contributions CLM and RMS both designed the study, analyzed data, and wrote/edited the manuscript. FS and TS gathered/ analyzed data and contributed toward writing/editing the manuscript.

Funding Research reported in this publication was supported in part by COVID Catalyst-I-3 Funds from the Woodruff Health Sciences Center and Emory School of Medicine and made possible through a grant from the O. Wayne Rollins Foundation, and through the Georgia CTSA NIH award (Grant No. UL1-TR002378). CLM is also supported by NIH/ NHLBI K99 HL150626-01.

Data availability The datasets generated during and analyzed during the current study are available from the corresponding author on reasonable request.

\section{Declarations}

Conflict of interest RMS has received research support from Cerus, Grifols, and is part of a scientific advisory board for OctaPharma. TS, FS, and CLM have no relevant disclosures.

Ethical approval This study was conducted under the oversight of the Emory University Institutional Review Board.

Informed consent The requirement for informed consent was waived by the Emory University Institutional Review Board.

\section{References}

1. Llitjos JF, Leclerc M, Chochois C, Monsallier JM, Ramakers M, Auvray M et al (2020) High incidence of venous thromboembolic events in anticoagulated severe COVID-19 patients. J Thromb Haemost 18(7):1743-1746

2. Yu B, Li X, Chen J, Ouyang M, Zhang H, Zhao X et al (2020) Evaluation of variation in D-dimer levels among COVID-19 and bacterial pneumonia: a retrospective analysis. J Thromb Thrombolysis 50(3):548-557

3. Panigada M, Bottino N, Tagliabue P, Grasselli G, Novembrino C, Chantarangkul V et al (2020) Hypercoagulability of COVID-19 patients in intensive care unit: a report of thromboelastography findings and other parameters of hemostasis. J Thromb Haemost 18(7):1738-1742

4. Spiezia L, Boscolo A, Poletto F, Cerruti L, Tiberio I, Campello E et al (2020) COVID-19-related severe hypercoagulability in patients admitted to intensive care unit for acute respiratory failure. Thromb Haemost 120(6):998-1000

5. Pavoni V, Gianesello L, Pazzi M, Stera C, Meconi T, Frigieri FC (2020) Evaluation of coagulation function by rotation thromboelastometry in critically ill patients with severe COVID-19 pneumonia. J Thromb Thrombolysis 50(2):281-286

6. Creel-Bulos C, Auld SC, Caridi-Scheible M, Barker N, Friend S, Gaddh $\mathrm{M}$ et al (2020) Fibrinolysis shutdown and thrombosis in A COVID-19 ICU. Shock (Augusta). https://doi.org/10.1097/SHK. 0000000000001635

7. Wright FL, Vogler TO, Moore EE, Moore HB, Wohlauer MV, Urban S et al (2020) Fibrinolysis shutdown correlation with thromboembolic events in severe COVID-19 infection. J Am Coll Surg 231(2):193-203.e1

8. Hardaway RM, Harke H, Tyroch AH, Williams CH, Vazquez Y, Krause GF (2001) Treatment of severe acute respiratory distress syndrome: a final report on a phase I study. Am Surg 67(4):377-382

9. Wang J, Hajizadeh N, Moore EE, McIntyre RC, Moore PK, Veress LA et al (2020) Tissue plasminogen activator (tPA) treatment for COVID-19 associated Acute Respiratory Distress Syndrome (ARDS): a case series. J Thromb Haemost. https://doi.org/10. $1111 /$ jth. 14828

10. Zmuda K, Neofotistos D, Ts'ao CH (2000) Effects of unfractionated heparin, low-molecular-weight heparin, and heparinoid on thromboelastographic assay of blood coagulation. Am J Clin Pathol 113(5):725-731

11. Schmidt AE, Israel AK, Refaai MA (2019) The utility of thromboelastography to guide blood product transfusion. Am J Clin Pathol 152(4):407-422

12. Mortus JR, Manek SE, Brubaker LS, Loor M, Cruz MA, Trautner BW et al (2020) Thromboelastographic results and hypercoagulability syndrome in patients with coronavirus disease 2019 who are critically ill. JAMA Netw Open 3(6):e2011192 
13. Liu F, Li L, Xu M, Wu J, Luo D, Zhu Y et al (2020) Prognostic value of interleukin-6, C-reactive protein, and procalcitonin in patients with COVID-19. J Clin Virol 127:104370

14. Moore HB, Moore EE, Chapman MP, Gonzalez E, Slaughter AL, Morton AP et al (2015) Viscoelastic measurements of platelet function, not fibrinogen function, predicts sensitivity to tissuetype plasminogen activator in trauma patients. J Thromb Haemost 13(10):1878-1887

15. Ostrowski SR, Haase N, Müller RB, Møller MH, Pott FC, Perner A et al (2015) Association between biomarkers of endothelial injury and hypocoagulability in patients with severe sepsis: a prospective study. Crit Care (London) 19(1):191

16. Bachler M, Bosch J, Sturzel DP, Hell T, Giebl A, Strohle M et al (2021) Impaired fibrinolysis in critically ill COVID-19 patients. Br J Anaesth 126(3):590-598

17. Jin X, Duan Y, Bao T, Gu J, Chen Y, Li Y et al (2020) The values of coagulation function in COVID-19 patients. PLoS ONE 15(10): 0241329

18. Kwaan HC (2020) Coronavirus disease 2019: the role of the fibrinolytic system from transmission to organ injury and sequelae. Semin Thromb Hemost 46(7):841-844

19. Dow N, Coleman JR, Moore H, Osborn ZT, Sackheim AM, Hennig $G$ et al (2020) Dense and dangerous: the tissue plasminogen activator-resistant fibrinolysis shutdown phenotype is due to abnormal fibrin polymerization. J Trauma Acute Care Surg 88(2):258-265

20. Ranucci M, Ballotta A, Di Dedda U, Bayshnikova E, Dei Poli M, Resta M et al (2020) The procoagulant pattern of patients with
COVID-19 acute respiratory distress syndrome. J Thromb Haemost 18(7):1747-1751

21. Faggiano P, Bonelli A, Paris S, Milesi G, Bisegna S, Bernardi N et al (2020) Acute pulmonary embolism in COVID-19 disease: preliminary report on seven patients. Int J Cardiol 313:129-131

22. Truong AD, Auld SC, Barker NA, Friend S, Wynn AT, Cobb $\mathrm{J}$ et al (2020) Therapeutic plasma exchange for COVID-19-associated hyperviscosity. Transfusion. https://doi.org/10.1111/trf. 16218

23. Weisel JW (2007) Structure of fibrin: impact on clot stability. J Thromb Haemost 5(Suppl 1):116-124

24. Manne BK, Denorme F, Middleton EA, Portier I, Rowley JW, Stubben $C$ et al (2020) Platelet gene expression and function in patients with COVID-19. Blood 136(11):1317-1329

25. Sahai A, Bhandari R, Koupenova M, Freedman J, Godwin M, McIntyre T, et al (2020) SARS-CoV-2 Receptors are expressed on human platelets and the effect of aspirin on clinical outcomes in COVID-19 patients. Res Sq. 2020

26. Akoluk A, Mazahir U, Douedi S, Aziz A, Obagi A, Kiss D et al (2020) Pulmonary embolism in COVID-19 treated with VA-ECLS and catheter tPA. Clin Med Insights 14:1179548420957451

Publisher's Note Springer Nature remains neutral with regard to jurisdictional claims in published maps and institutional affiliations. 